\title{
Inference and Properties of Mixture Two Extreme Lower Bound Distributions
}

\author{
Fathy H. Riad ${ }^{1,2}$ \\ ${ }^{1}$ Department of Mathematics, Faculty of Science, Minia University, Minya, Egypt \\ ${ }^{2}$ Department of Mathematics, Faculty of Sciences, Aljouf University, Sakakah, KSA \\ Email: fathyhamdi2008@yahoo.com
}

How to cite this paper: Riad, F.H. (2017) Inference and Properties of Mixture Two Extreme Lower Bound Distributions. Engineering, 9, 517-523.

https://doi.org/10.4236/eng.2017.96032

Received: May 3, 2017

Accepted: June 18, 2017

Published: June 21, 2017

Copyright (c) 2017 by author and Scientific Research Publishing Inc. This work is licensed under the Creative Commons Attribution International License (CC BY 4.0).

http://creativecommons.org/licenses/by/4.0/

\begin{abstract}
In this paper, we discuss the mixture model of two extreme lower bound distributions. First, some properties we obtain of the model with hazard function are discussed. In addition, the estimates of the unknown parameters via the EM algorithm are obtained. The performance of the findings in the paper is showed by demonstrating some numerical illustrations through Monte Carlo simulation.
\end{abstract}

\section{Keywords}

Mixture Extreme Lower Bound Distribution, Reliability, Estimation, EM Algorithm, Monte Carlo Simulation

\section{Introduction}

Recently, the extreme value distribution is becoming increasingly important in engineering statistics as a suitable model to represent phenomena with usually large maximum observations. In engineering circles, this distribution is often called the extreme lower bound model. It is one of the pioneers of extreme value statistics. The extreme lower bound distribution is one of the probability distributions used to model extreme events. The generalization of the standard extreme lower bound distribution has been introduced by Nadarajah and Kotz [1] and Abd-Elfattah [2]. There are over fifty applications ranging from accelerated life testing through to earthquakes, floods, rain fall, queues in supermarkets, sea currents, wind speeds and track race records, see Kotz and Nadarajah [3]. Mixture models play an important role in many practical applications. For example, direct applications of finite mixture models are in fisheries research, economics, medicine, psychology, palaeoanthropology, botany, agriculture, zoology, life testing and reliability. Direct applications 
include outliers, Gaussian sums, cluster analysis, latent structure models, modeling prior densities, empirical Bayes method and nonparametric density estimation. In many applications, the available data can be considered as data coming from a mixture population of two or more distributions. This data enables us to mix statistical distributions to get a new distribution which has the properties of its components. For an excellent survey of estimation techniques, discussion and applications, mixture distribution have been considered extensively by many authors, see Titterington [4], Maclachlan and Basford [5], Lindsay [6], Maclachlan and Krishnan [7] and Maclachlan and Peel [8]. Recently, there are many authors [4] [9] [10] who discuss the mixture models, Mohie El-Din et al. [11] [12] [13]. In this paper, we discuss some important measures of two extreme lower bound distributions. Also, we estimate the vector of unknown parameters of a mixture model via the EM algorithm proposed by Dempster et al. [9]. Further, we carry out some simulated illustrations using Monte Carlo method.

\section{Description of the Model}

The mixture of two extreme lower bound distributions has its pdf as

$$
f(x, \Theta)=H_{1} f_{1}\left(x, \Theta_{1}\right)+H_{2} f_{2}\left(x, \Theta_{2}\right) \quad: H_{1}+H_{2}=1
$$

where $\Theta=\left(H_{1}, \Theta_{j}\right), \Theta_{j}=\left(c_{j}, \beta_{j}, \theta_{j}\right), j=1,2$ and $f\left(x, \Theta_{j}\right)$, the density function of $j \underline{t h}$ component, is given by

$$
f_{j}(x)=\left(\frac{c_{j}}{\beta_{j}}\right)\left(\frac{x-\theta_{j}}{\beta_{j}}\right)^{-c_{j}-1} \exp \left[-\left(\frac{x-\theta_{j}}{\beta_{j}}\right)^{-c_{j}}\right], \quad(x>\theta, c>0, \beta>0) .
$$

The $c d f$ of the mixture of two extreme lower bound distributions is given by

$$
F(x, \Theta)=H_{1} F_{1}\left(x, \Theta_{1}\right)+H_{2} F_{2}\left(x, \Theta_{2}\right) \quad: H_{1}+H_{2}=1
$$

where $F_{j}(x, \Theta)$, the $c d f$ th $\underline{h}$ component, is given by

$$
F_{j}(x)=\exp \left[-\left(\frac{x-\theta_{j}}{\beta_{j}}\right)^{-c_{j}}\right], \quad(x>\theta, c>0, \beta>0) .
$$

Such that, We study this case, when $C$ and $\beta$ are the parameters unknown and $\theta$ is the parameter known.

\section{Properties}

In this section we obtain some properties for two extreme lower bound distribution by extending the corresponding results of the two parameters extreme lower bound distribution where (2.3) $\theta$ is known as follow.

\subsection{The Expected Value and Variance}

The expected value of the $p d f$ of the two extreme lower bound distribution obtain in (2.1) and (2.3) is 


$$
E(x)=H_{1} \beta_{1} \Gamma\left(1-\frac{1}{c_{1}}\right)+H_{2} \beta_{2} \Gamma\left(1-\frac{1}{c_{2}}\right)
$$

and the variance is given by

$$
\begin{aligned}
\operatorname{Var}(x)= & H_{1} \beta_{1}^{2}\left[\Gamma\left(1-\frac{2}{c_{1}}\right)-H_{1} \Gamma^{2}\left(1-\frac{1}{c_{1}}\right)\right] \\
& +H_{2} \beta_{2}^{2}\left[\Gamma\left(1-\frac{2}{c_{2}}\right)-H_{2} \Gamma^{2}\left(1-\frac{1}{c_{2}}\right)\right] \\
& -2 H_{1} H_{2} \beta_{1} \beta_{2} \Gamma\left(1-\frac{1}{c_{1}}\right) \Gamma\left(1-\frac{1}{c_{2}}\right)
\end{aligned}
$$

\subsection{Mode and Median}

The mode of the mixture of two extreme lower bound distribution is obtained by solving the following nonlinear equation with respect to $x$

$$
\sum_{i=0}^{2} H_{j}\left(\frac{x-\theta_{j}}{\beta_{j}}\right)^{-\left(2+c_{j}\right)} \mathrm{e}^{-\left(\frac{x-\theta_{j}}{\beta_{j}}\right)^{-c_{j}}}\left[-\left(1+c_{j}\right)+c_{j}\left(\frac{x-\theta_{j}}{\beta_{j}}\right)^{-c_{j}}\right]
$$

By using (2.4) and (3.5), the median of the mixture of two extreme lower bound distribution is obtained by solving the following nonlinear equation with respect to $X$

$$
H_{j} \mathrm{e}^{-\left(\frac{x-\theta_{j}}{\beta_{1}}\right)^{-c_{1}}}+H_{j} \mathrm{e}^{-\left(\frac{x-\theta_{j}}{\beta_{2}}\right)^{-c_{2}}}=0.5
$$

From Table 1, we obtain the median and the mode of the mixture two extreme lower bound distribution based on different choices of the parameters $H_{i}$ and $\Theta_{j}$ for each $j=1,2$ from this table we observe that the mode is slightly affected by the variation in the values of the mixing proportion $H_{1}$, while one mode is stable in the bimodal case. In addition, for unimodal case, the median increases when $H_{1}$ increases. From the bimodal case, we observe that the median decreases when $H_{1}$ icreases.

\subsection{Reliability and Failure Rate Function}

The reliability function of the mixture two extreme lower bound distribution is given by

Table 1. The median and the mode of the mixture of two extreme lower bound distribution.

\begin{tabular}{cccc}
\hline Bimodel case & & \\
\hline$\Theta=\left(H_{1}, C_{1}, \beta_{2}, c_{2}, \beta_{2}\right)$ & $(0.2,2.5,2,1,2.9)$ & $(0.4,2.5,2,1,2.9)$ & $(0.6,2.5,2,1,2.9)$ \\
\hline Median & 1.0286 & 0.9201 & 0.7852 \\
Mode & $0.326,0.885$ & $0.326,0.863$ & $0.326,0.798$ \\
\hline Unimodel case & & & \\
\hline$\Theta=\left(H_{1}, c_{1}, \beta_{2}, c_{2}, \beta_{2}\right)$ & $(0.2,1,2,2,3)$ & $(0.4,1,2,2,3)$ & $(0.6,1,2,2,3)$ \\
\hline Median & 0.635 & 0.733 & 0.882 \\
Mode & 0.459 & 0.469 & 0.882
\end{tabular}




$$
R(x)=H_{1}\left[1-\mathrm{e}^{-\left(\frac{x-\theta_{1}}{\beta_{1}}\right)^{-c_{1}}}\right]+H_{2} l\left[1-\mathrm{e}^{-\left(\frac{x-\theta_{2}}{\beta_{2}}\right)^{-c_{2}}}\right]
$$

By using (2.4) and (3.5) it can be seen the failure rate function (hazard rate function) of the mixture two extreme lower bound distribution is given by

$$
r(x)=\frac{H_{1} \frac{c_{1}}{\beta_{1}}\left(\frac{x-\theta_{1}}{\beta_{1}}\right)^{-\left(1+c_{1}\right)} \mathrm{e}^{-\left(\frac{x-\theta_{2}}{\beta_{1}}\right)^{-c_{1}}}+H_{2} \frac{c_{2}}{\beta_{2}}\left(\frac{x}{\beta_{2}}\right)^{-\left(1+c_{2}\right)} \mathrm{e}^{-\left(\frac{x}{\beta_{2}}\right)^{-c_{2}}}}{R(x)} .
$$

Which can be written as

$$
r(x)=k(x) r_{1}(x)+(1-k(x)) r_{2}(x)
$$

where

$$
k(x)=\frac{H_{1} R_{1}(x)}{H_{1} R_{1}(x)+H_{2} R_{2}(x)}, r_{j}(x)=\frac{f_{j}(x)}{R_{j}(x)}
$$

and

$$
R_{j}(x)=1-\mathrm{e}^{-\left(\frac{x-\theta_{j}}{\beta_{1}}\right)^{-c_{1}}}
$$

The failure rate function of the mixture two extreme lower bound distribution given in (3.10) satisfies the following limits

$$
\lim _{x \rightarrow \infty} r(x)=0 \text { and } \lim _{x \rightarrow 0} r(x)=0
$$

\section{Estimation via EM Algorithm}

The EM algorithm provides a simple computational method for fitting mixture models. We use the EM algorithm to estimate the parameters of the pdf of the mixture two extreme lower bound distribution which given in (2.1) and (2.3). We focus in this section, the Maximum likelihood fitting of two extreme lower bound distributions mixture via the EM algorithm. Maclachlan and Peel [9], the essential nature of the algorithm is the alternation of expectation and maximization steps.

$$
\begin{aligned}
& \xi_{1}=\sum_{j=1}^{n}\left[\frac{H_{j}^{m}\left(\frac{y_{j}-\theta_{j}}{\beta_{j}}\right)^{-\left(1+c_{j}\right)} \mathrm{e}^{\left(\frac{y_{j}-\theta_{j}}{\beta_{j}}\right)^{-c_{j}}}}{\left.\sum_{j=1}^{2} H_{j}^{m}\left(\frac{y_{j}-\theta_{j}}{\beta_{j}}\right)^{-\left(1+c_{j}\right)} \mathrm{e}^{\left(\frac{y_{j}-\theta_{j}}{\beta_{j}}\right)^{-c_{j}}}\right]}\right. \\
& \xi_{2}=\sum_{j=1}^{n}\left[\frac{H_{j}^{m} \beta_{j}^{c_{j}}\left(\frac{y_{j}-\theta_{j}}{\beta_{j}}\right)^{-\left(1+2 c_{j}\right)} \mathrm{e}^{\left(\frac{y_{j}-\theta_{j}}{\beta_{j}}\right)^{-c_{j}}}}{\left.\sum_{j=1}^{2} H_{j}^{m}\left(\frac{y_{j}-\theta_{j}}{\beta_{j}}\right)^{-\left(1+c_{j}\right)} \mathrm{e}^{\left(\frac{y_{j}-\theta_{j}}{\beta_{j}}\right)^{-c_{j}}}\right]}\right]
\end{aligned}
$$

then, Concerning the E-step on the $(m+1)$ iteration, the updated estimate of 
the $j$ mixing proportion $H_{j}$ is given by

$$
H_{j}^{(m+1)}=\frac{1}{n} \xi_{1}
$$

From (4.13) we obtain the M-step of the $(m+1)$ iteration, the updated estimates $c_{j}^{(m+1)}$ and $\beta_{j}^{(m+1)}$ for each $j=1,2$ are obtained, respectively, by solving the following systems of equations

$$
C_{j}^{(m+1)}=\left(\frac{\xi_{1}}{\xi_{2}}\right)^{-\frac{1}{c_{j}}}
$$

and

$$
\sum_{j=1}^{n} T_{j}\left[\frac{1}{c_{j}}+\log \beta_{j}-\log \left(y_{j}-\theta_{j}\right)+\left(\frac{y_{j}-\theta_{j}}{\beta_{j}}\right)^{-c_{j}} \log \left(\frac{y_{j}-\theta_{j}}{\beta_{j}}\right)\right]=0
$$

where

$$
T_{j}=\frac{H_{j}^{m}\left(\frac{y_{j}-\theta_{j}}{\beta_{j}}\right)^{-\left(1+c_{j}\right)} \mathrm{e}^{-\left(\frac{y_{j}-\theta_{j}}{\beta_{j}}\right)^{-c_{j}}}}{\sum_{j=1}^{2} H_{j}^{m}\left(\frac{y_{j}-\theta_{j}}{\beta_{j}}\right)^{-\left(1+c_{j}\right)} \mathrm{e}^{-\left(\frac{y_{j}-\theta_{j}}{\beta_{j}}\right)^{-c_{j}}}, \quad j=1,2 \text { and } H_{1}+H_{2}=1 .}
$$

The estimates of $H, c_{1}, \beta_{1}, c_{2}$ and $\beta_{2}$ are obtained by solving (4.15), (4.17) and (4.18). Equations (4.15) and (4.17) are written explicitly but Equation (4.18) has to be solved numerically with random choices of the initial values.

\section{Numerical Illustration}

In order to calculate the estimates of the five parameters $H, c_{1}, \beta_{1}, c_{2}$ and $\beta_{2}$ where $\theta_{1}$ and $\theta_{2}$ are known that appear in the pdf of the mixture two extreme lower bound distribution given in (2.1) and (2.3) by using EM algorithm in a Monte Carlo simulation as follows:

Generate random sample of size $n=50$ and 100 from the mixture two extreme lower bound distribution distribution with for each choice of the parameters $H, c_{1}, \beta_{1}, \theta_{1}, c_{2}, \theta_{2}$ and $\beta_{2}$. Some of choices caver the unimodal case and other caver the bimodal case.

The random samples of the mixtures are generated with respect to two uniform variables $u_{1}$ and $u_{2}$. If $u_{1}<H_{1}$, then use $u_{2}$ to generate a random variable $x$ from the mixture two extreme lower bound distribution by using (3.5) as $x=F_{1}^{-1}\left(u_{2}\right)$, but if $u_{1}<H_{1}$, then $x=F_{2}^{-1}\left(u_{2}\right)$.

The bias and the mean square errors of the estimates are calculated based on 10,000 Monte Carlo simulation and the results are illustrated in Table 2 and Table 3. We see that in most of the considered cases, the mean square errors of the estimated parameters decrease as $n$ increase.

\section{Conclusion}

In this paper, the behaviors of the mode and median of the mixture two extreme 
Table 2. Bais of the estimate of $\hat{\Theta}$ based on EM algorithm.

\begin{tabular}{|c|c|c|c|c|c|c|}
\hline Bimodal & & & & & & \\
\hline$\Theta=\left(H_{1}, c_{1}, \beta_{1}, c_{2}, \beta_{2}\right)$ & $n$ & $\hat{H}_{1}$ & $\hat{c}_{1}$ & $\hat{\beta}_{1}$ & $\hat{c}_{2}$ & $\hat{\beta}_{2}$ \\
\hline \multirow[t]{2}{*}{$(0.2,2.5,2,1,2.9)$} & 50 & -0.116 & -1.204 & -0.313 & 0.299 & -1.203 \\
\hline & 100 & -0.056 & -1.189 & -0.333 & 0.313 & -1.242 \\
\hline \multirow[t]{2}{*}{$(0.4,2.5,2,1,2.9)$} & 50 & -0.068 & -0.770 & -0.411 & 0.729 & -1.309 \\
\hline & 100 & -0.001 & -0.757 & -0.421 & 0.743 & -1.333 \\
\hline \multirow[t]{2}{*}{$(0.6,2.5,2,1,2.9)$} & 50 & 0.006 & -0.614 & -0.398 & 0.894 & -1.270 \\
\hline & 100 & 0.015 & -0.611 & -0.379 & 0.886 & -1.295 \\
\hline \multicolumn{7}{|l|}{ Unimodal } \\
\hline$\Theta=\left(H_{1}, c_{1}, \beta_{1}, c_{2}, \beta_{2}\right)$ & $n$ & $\hat{H}_{1}$ & $\hat{c}_{1}$ & $\hat{\beta}_{1}$ & $\hat{c}_{2}$ & $\hat{\beta}_{2}$ \\
\hline \multirow[t]{2}{*}{$(0.2,1,2,2,3)$} & 50 & -0.042 & 0.864 & 0.487 & -0.136 & -0.514 \\
\hline & 100 & -0.025 & 0.850 & 0.423 & -0.131 & -0.542 \\
\hline \multirow[t]{2}{*}{$(0.4,1,2,2,3)$} & 50 & 0.011 & 0.565 & -0.019 & -0.483 & -1.025 \\
\hline & 100 & 0.005 & 0.569 & -0.015 & -0.438 & -0.101 \\
\hline \multirow[t]{2}{*}{$(0.6,1,2,2,3)$} & 50 & 0.024 & 0.465 & -0.089 & -0.613 & -1.170 \\
\hline & 100 & 0.006 & 0.494 & -0.074 & -0.573 & -1.132 \\
\hline
\end{tabular}

Table 3. MSE of $\hat{\Theta}$ based on EM algorithm.

\begin{tabular}{|c|c|c|c|c|c|c|}
\hline Unimodal & & & & & & \\
\hline$\Theta=\left(H_{1}, c_{1}, \beta_{1}, c_{2}, \beta_{2}\right)$ & $n$ & $\hat{H}_{1}$ & $\hat{c}_{1}$ & $\hat{\beta}_{1}$ & $\hat{c}_{2}$ & $\hat{\beta}_{2}$ \\
\hline \multirow[t]{2}{*}{$(0.2,2.5,2,1,2.9)$} & 50 & 0.025 & 1.0002 & 0.1003 & 0.098 & 1.003 \\
\hline & 100 & 0.006 & 1.0001 & 0.120 & 0.095 & 1.0001 \\
\hline \multirow[t]{2}{*}{$(0.4,2.5,2,1,2.9)$} & 50 & 0.202 & 1.127 & 0.1003 & 0.200 & 2.005 \\
\hline & 100 & 0.309 & 1.0007 & 0.1001 & 0.199 & 2.002 \\
\hline \multirow[t]{2}{*}{$(0.6,2.5,2,1,2.9)$} & 50 & 0.002 & 0.377 & 0.108 & 0.798 & 2.002 \\
\hline & 100 & 0.0005 & 0.369 & 0.1008 & 0.789 & 2.000 \\
\hline \multicolumn{7}{|l|}{ Unimodal } \\
\hline$\Theta=\left(H_{1}, c_{1}, \beta_{1}, c_{2}, \beta_{2}\right)$ & $n$ & $\hat{H}_{1}$ & $\hat{c}_{1}$ & $\hat{\beta}_{1}$ & $\hat{c}_{2}$ & $\hat{\beta}_{2}$ \\
\hline \multirow[t]{2}{*}{$(0.2,1,2,2,3)$} & 50 & 0.0021 & 0.7002 & 0.2384 & 0.0189 & 0.2005 \\
\hline & 100 & 0.0005 & 0.7003 & 0.1798 & 0.0183 & 0.2004 \\
\hline \multirow[t]{2}{*}{$(0.4,1,2,2,3)$} & 50 & 0.0084 & 0.5987 & 0.0725 & 0.0482 & 0.5000 \\
\hline & 100 & 0.0024 & 0.5169 & 0.0248 & 0.0430 & 0.5023 \\
\hline \multirow[t]{2}{*}{$(0.6,1,2,2,3)$} & 50 & 0.0020 & 0.2145 & 0.0083 & 0.3001 & 1.000 \\
\hline & 100 & 0.0002 & 0.2003 & 0.0061 & 0.3000 & 0.9998 \\
\hline
\end{tabular}

lower bound distribution are investigated, based on different choices of the parameters. Also, the behaviors of the failure rate function are discussed through some different graphs. In addition, the estimation of the unknown parameters is obtained using the EM algorithm. Finally, a Monte Carlo simulation based on 10,000 runs is carried out. 


\section{References}

[1] Abd-Elfattah, A.M. and Omima, A.M. (2009) Estimation of the Unknown Parameters of the Generalized Frechet Distribution. Journal of Applied Sciences Research, 5, 1398-1408.

[2] Dempster, A. P., Laird, N. M., Rubin, D. B. (1977) Maximum-Likelihood from Incomplete Data via the EM Algorithm. Journal of the Royal Statistical Society. Series $B$ (Methodological), 39, 1-38.

[3] Everitt, B.S. and Hand, D.J. (1981) Finite Mixture Distribution. Chapman and Hall, London. https://doi.org/10.1007/978-94-009-5897-5

[4] Jiang, R., Zuo, M.J. and Li, H. (1999) Weibull and Inverse Weibull Mixture Models Allowing Negative Weights. Reliability Engineering \& System Safety, 66, 227-234.

[5] Kotz, S. and Nadarajah, S. (2000) Extreme Value Distributions: Theory and Applications. Imperial College Press, London. https://doi.org/10.1142/p191

[6] Landsay, B.G. (1995) Mixture Models: Theory, Geometry and Applications. The Institute of Mathematical Statistics, Hayward, CA.

[7] Maclachlan, G.J. and Basford, K.E. (1988) Mixture Models: Applications to Clustering. Marcel Dekker, New York.

[8] Maclachlan, G.J. and Krishnan, T. (1997) The EM Algorithm and Extensions. Wiley, New York.

[9] Maclachlan, G.J. and Peel, D. (2000) Finite Mixture Models. Wiley, New York. https://doi.org/10.1002/0471721182

[10] Mohie El-Din, M.M., Abdel-Aty, Y. and Riad, F.H. (2010) Estimations of the Parameters of the Extreme Lower Bound Distribution with Progressively Censored Data. Far East Journal of Theoretical Statistics, 32, 25-34.

[11] Mohie El-Din, M.M., Riad, F.H. and El-Sayed, M.A. (2014) Statistical Inference and Prediction for the Inverse Weibull Distribution Based on Record Data. Journal of Statistics Applications and Probability, 3, 171-177. https://doi.org/10.12785/jsap/030207

[12] Mohie El-Din, M.M., Riad, F.H. and El-Sayed, M.A. (2014) Confidence Intervals for Parameters of IWD Based on MLE and Bootstrap. International Journal of Advanced Statistics and Probability, 2, 28-33.

[13] Nadarajah, S. and Kotz, S. (2003) The Exponentiated Frechet Distribution. Interstat Lectronic Journal.

\section{Submit or recommend next manuscript to SCIRP and we will provide best service for you:}

Accepting pre-submission inquiries through Email, Facebook, LinkedIn, Twitter, etc. A wide selection of journals (inclusive of 9 subjects, more than 200 journals)

Providing 24-hour high-quality service

User-friendly online submission system

Fair and swift peer-review system

Efficient typesetting and proofreading procedure

Display of the result of downloads and visits, as well as the number of cited articles

Maximum dissemination of your research work

Submit your manuscript at: http://papersubmission.scirp.org/

Or contact eng@scirp.org 